\title{
Live imaging of NPCs
}

\section{By Tim Fulmer, Staff Writer}

Noninvasive imaging of neuronal progenitor cells in the live human brain has been impossible because of the shortcomings of existing imaging methods, specifically the toxicity of PET and the low sensitivity associated with MRI. A new method based on a high-sensitivity form of proton magnetic resonance spectroscopy ( ${ }^{1} \mathrm{H}$-MRS) could avoid both drawbacks and noninvasively detect endogenous CNS biomarkers in the human brain.

Scientists from the State University of New York at Stony Brook, Cold Spring Harbor Laboratory and Brookhaven National Laboratory reported in the Nov. 9, 2007 issue of Science ${ }^{1}$ that ${ }^{1} \mathrm{H}-\mathrm{MRS}$ identified a 1.28-p.p.m. spectral peak that was significantly increased in neuronal progenitor cells (NPCs) compared with the same peak in neurons and glial cells. Although the molecule or molecules corresponding to the 1.28-p.p.m. peak still must be characterized, initial data demonstrated the technology's ability to identify and track NPCs in rat and human brains.

NPCs are responsible for neurogenesis, a cell proliferation and maturation process that persists throughout adulthood primarily in two regions of the brain: the dentate gyrate (DG) of the hippocampus and the subventricular zone (SVZ).

Aberrant neurogenesis in the hippocampus has been implicated in psychiatric disorders such as depression ${ }^{2}$ and neurodegenerative conditions including Alzheimer's disease. ${ }^{3}$ Also, ischemic and traumatic brain lesions are known to affect neurogenesis in both the DG and the SVZ. ${ }^{4}$ Moreover, NPCs implanted in the rodent CNS are capable of integrating with gliomas and could therefore potentially be used to track cancer progression or deliver tumor-specific therapies. ${ }^{5}$

Grigori Enikolopov, associate professor of neurology at Cold Spring Harbor and a corresponding author of the Science paper, said he believes the new technique could be used not only in a diagnostic setting to monitor psychiatric disorders, neurodegenerative diseases and brain cancers, but also as a drug discovery tool for evaluating potential therapies that target regions of the brain enriched with NPCs.

On the drug discovery front, Carrolee Barlow, CSO of BrainCells Inc., told SciBX that the company is interested in using the method as a "surrogate measure of neurogenesis" in the upcoming Phase II trials of BCI-540 to treat depression.

BrainCells uses a combination of in vitro assays and animal models to screen for compounds that stimulate neurogenesis. ${ }^{6}$ The mechanism of lead compound BCI-540 is undisclosed.
BrainCells also is using its neurogenesis-based in vitro and in vivo assays to screen CNS targets and compounds for $\mathbf{H}$. Lundbeck $\mathbf{A} / \mathbf{S}$.

Neither Barlow nor the researchers would disclose whether they are in discussions for a potential licensing deal, but Barlow did say the method fits well with the company's screening strategy.

Mirjana Maletic-Savatic, assistant professor of neurology at SUNY Stony Brook and another corresponding author of the paper, said she is now collaborating with Tarique Perera and Scott Small, both of Columbia University, to use the method for long-term monitoring of the effects of antidepressants on NPCs in patients and healthy volunteers.

Data from a number of preclinical studies suggest that reduced hippocampal neurogenesis contributes to the pathophysiology of depression and that reversing or preventing decreased neurogenesis could be at least one way by which antidepressants work. In 2007, for example, Perera published the first data demonstrating that antidepressant therapy increases neurogenesis in the nonhuman primate hippocampus. ${ }^{7}$ Also, a number of antidepressant drugs, including the selective serotonin reuptake inhibitor Prozac fluoxetine from Eli Lilly and Co. and the monoamine oxidase inhibitor Parnate tranylcypromine from GlaxoSmithKline plc, were previously shown to increase neurogenesis in nonprimate animal models. ${ }^{2}$

According to Maletic-Savatic, the method requires 45-60 minutes for data collection and another day for analysis. Further refinements in the signal acquisition algorithm might eventually allow imaging of neurogenesis in the SVZ, where the signal is not easily extracted from the background noise of nearby cerebrospinal fluid.

The SUNY researchers are also working to determine the chemical nature of the 1.28-p.p.m. peak. Initial spectroscopic characterization has revealed that the biomarker is hydrophobic and could be a mixture of saturated and monounsaturated fatty acids.

\section{REFERENCES}

1. Manganas, L.N. et al. Science 318, 980-985 (2007)

Contact: Grigori Enikolopov, Cold Spring Harbor National Laboratory,

Cold Spring Harbor, N.Y.

e-mail: enikolop@cshl.edu

Contact: Mirjana Maletic-Savatic, Department of Neurology, SUNY Stony Brook, Stony Brook, N.Y.

e-mail: mmaleticsava@notes.cc.sunysb.edu

2. Malberg, J.E. J. Psychiatry Neurosci. 29, 196-205 (2004)

3. Jin, K. et al. Proc. Natl. Acad. Sci. USA 101, 343-347 (2004)

4. Johansson, B.B. J. Cereb. Blood Flow Met. 27, 1417-1430 (2007)

5. Shah, K. \& Weissleder, R. NeuroRx 2, 215-225 (2005)

6. Truong, R. BioCentury 14(37), 11-12; Aug. 21, 2006

7. Perera, T.D. et al. J. Neurosci. 27, 4894-4901 (2007)

\section{COMPANIES AND RESEARCH INSTITUTIONS MENTIONED}

BrainCells Inc., San Diego, Calif.

Brookhaven National Laboratory, Brookhaven, N.Y.

Cold Spring Harbor Laboratory, Cold Spring Harbor, N.Y.

Columbia University, New York, N.Y.

Eli Lilly and Co. (NYSE:LLY), Indianapolis, Ind.

GlaxoSmithKline plc (LSE:GSK; NYSE:GSK), London, U.K.

H. Lundbeck A/S (CSE:LUN), Copenhagen, Denmark

State University of New York at Stony Brook, Stony Brook, N.Y. 